\title{
Transverse electron guns for plasma excitation
}

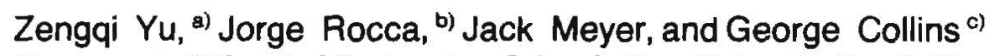 \\ Department of Electrical Engineering, Colorado State University. Fort Collins, Colorado 80523
}

\begin{abstract}
(Received 9 October 1981; accepted for publication 10 December 1981)
We report a plasma gun, which generates on a continuous basis, kilowatt electron beam discharges (e.g., $0.4 \mathrm{~A}$ at $4 \mathrm{kV}$ ) in an ambient pressure 0.1-1 Torr without differential pumping.

Gun design characteristics, operating parameters, and measured beam profiles are given. Electron beam generation on a pulsed basis has also been studied. More than $100 \mathrm{~A}$ of beam current has been measured with a Faraday cup biased to $-100 \mathrm{~V}$. A trapped electron beam scheme for achieving efficient deposition of the electron beam energy in a gas medium is described.
\end{abstract}

PACS numbers: 52.50.Dg, 52.80.Hc

\section{INTRODUCTION}

Hollow cathode devices have already been used to generate electron beams in the $1-1000 \mu$ pressure region with beam generation efficiencies from 60 to $70 \%{ }^{1,2}$ By way of comparison, the operation of conventional, hot filament type, electron guns is restricted to ambient pressures below $10^{-4}$ Torr. The operating pressure of these hollow cathode devices makes them attractive for pumping continuouswave (cw) ion lasers ${ }^{3}$, processing of material surfaces, ${ }^{4}$ for plasma excitation, and as ionization sources in broad beam ion sources.

Electron beams operating at a voltage near one $\mathrm{kV}$ when directed into a gas can efficiently create multiply ionized species ${ }^{5}$ because of the close match of the electron energy with the peaks in the ionization cross sections. Multiply ionized species have a rich spectrum in the ultraviolet (UV) and vacuum ultraviolet (VUV) because of the large energy spacing between energy levels. In conventional gas discharges, such species are only weakly populated, primarily because the electron energy distribution has few electrons with sufficient energy. In order to increase the average electron energy, operation of pulsed gas discharges at low pressures has usually been attempted. This scheme has the disadvantage of low operating efficiency and moreover, operation is usually restricted to a pulsed basis only. Our long term goal is to deposit electron beam energy into a gas and thereby provide a novel discharge in which intense VUV radiation may be generated at high efficiency.

In this paper we present our gun designs and their experimentally determined operating characteristics as well as the spatial profile of the generated electron beam. We have developed electron guns operating from $1-10 \mathrm{kV}$ in ambient pressures of 0.1-1.0 Torr. These guns operate continuously at the multi-kilowatt level and at $>80 \mathrm{~kW}$ on a pulsed basis. We suggest a trapped beam mode of operation for the purpose of enhanced efficiency of energy deposition of the electron beam into a gaseous medium.

\footnotetext{
${ }^{2}$ Permanent Address: Dept. of Physics, Fudan University, Shanghai, P. R. C.

${ }^{b}$ Spectra-Physics Industrial Fellow.

c)Alfred Sloan Foundation Fellow 1979-81.
}

\section{HOLLOW CATHODE TRANSVERSE ELECTRON GUN DESIGN AND OPERATION}

High purity graphite was chosen as the cathode material due to its low sputtering yield. ${ }^{6}$ All of the guns had a 20$\mathrm{cm}$-long active region. To avoid external electron emission, the graphite cathodes were surrounded with insulating ceramic tubes $\left(99.8 \% \mathrm{Al}_{2} \mathrm{O}_{3}\right)$ with approximately a $1-\mathrm{mm}$ separation between the graphite and ceramic. See Fig. 1. The graphite body was made with a large $(2.9-\mathrm{cm})$ diameter so that operation in a dc mode at a moderate power level $(1 \mathrm{~kW}$ of input power) could be achieved without water cooling. In this case, the gun is radiation cooled. When multi-kilowatt $\mathrm{dc}$ operation is required, water cooling can be implemented, as shown in Fig. 1, by running water through two metal tubes that fit within the graphite core of the guns. In one of the tests, the front wall of the graphite was shaped to be concave with a $1.5-\mathrm{cm}$ radius of curvature to increase the electron density in front of the cathode by focusing the electron beam. See the cathode face in Fig. $1(\mathrm{~b})$ and the corresponding plasma in Fig. 1(c). Different slot widths for the slotted graphite nose have been tested $(2.5,5$, and $7 \mathrm{~mm})$ and their differing characteristics are compared below. In all cases the total width of the graphite nose was $1.25 \mathrm{~cm}$. It was also observed that slot depths of 1.9 and $3.2 \mathrm{~cm}$ had the same discharge characteristics.

These electron guns have two modes of operation. One mode is a high impedance one in which an electron beam is produced, and the other is a low impedance mode where no electron beam is produced. An internal plasma created within the slot works as a virtual cathode for the electron beam in the following manner. Electrons emitted by the internal walls of the cathode, as a result of ion bombardment and photoelectric emission, are accelerated through an internal hollow cathode voltage drop of a few hundred eV. These electrons are electrostatically trapped inside the slot and lose most of their energy in ionizing and exciting collisions that sustain the internal plasma. In the high impedance mode, an external dark space also exists, which presents a voltage drop of several $\mathrm{kV}$ to the electrons which emerge from the hollow cathode plasma. Under the above conditions, an electron beam is created. An additional portion of the electron beam emission is produced by ions that bombard the cathode front 


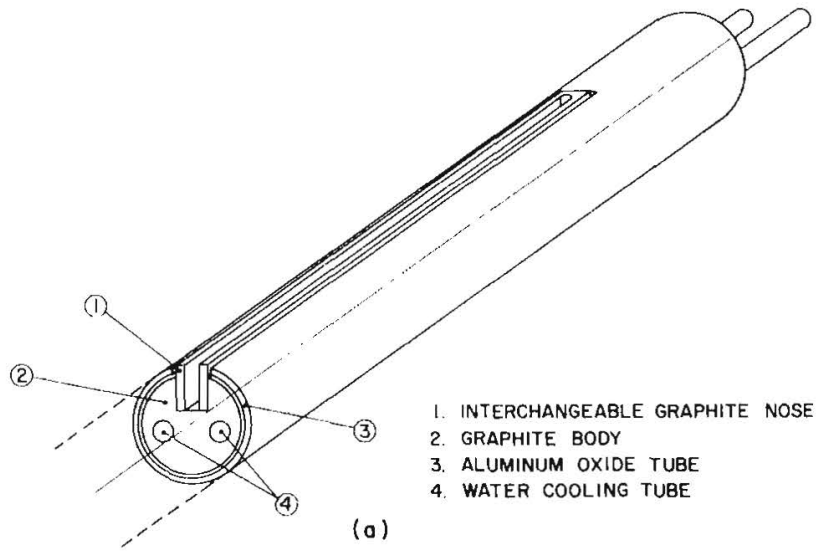

(o)

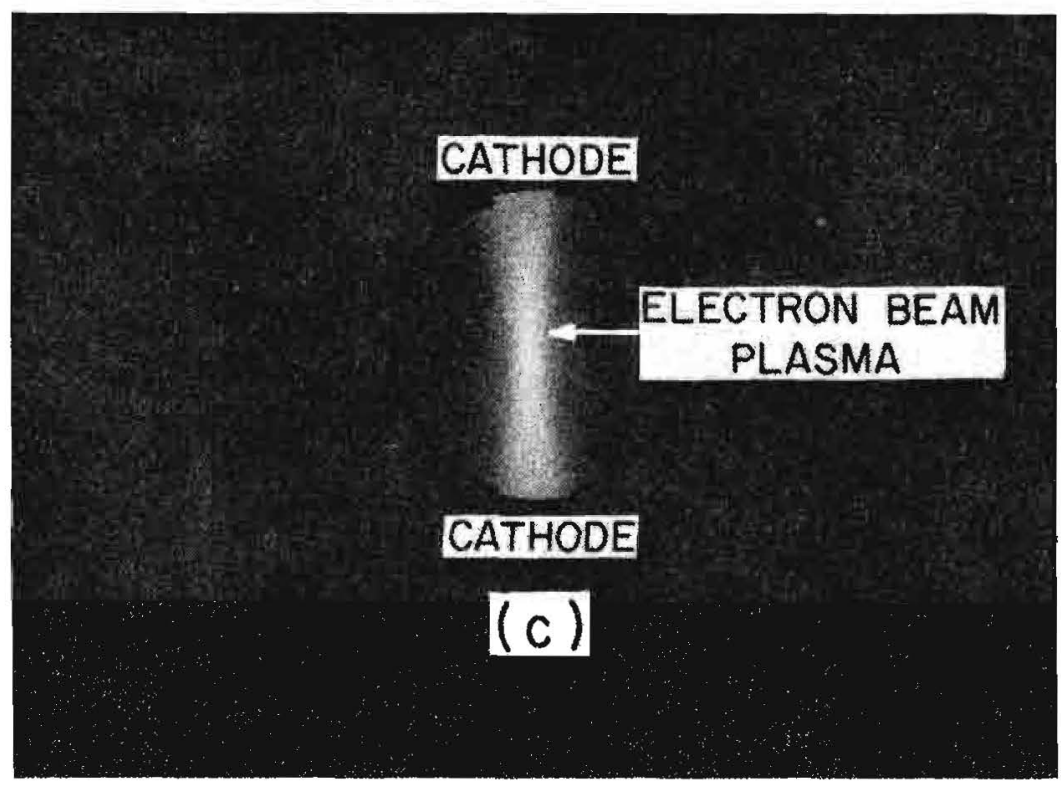

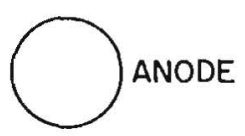

EXTERNAL

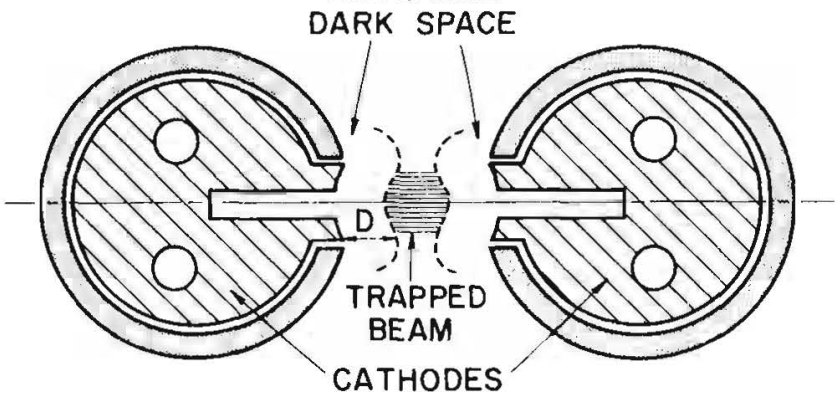

(b) wall and create secondary electrons that are subsequently accelerated through the same external dark space. The electron beam discharge is shown in Fig. 2. Operation in the beam mode occurs over a limited range of current and pressure. When these two parameters are not properly chosen the external dark space vanishes, production of the electron beam ceases, and the discharge then operates in the low impedance mode. In this operating mode, the voltage drop is a few hundred $V$ and a positive column occupies the distance between the electrodes. This transition from beam to low impedance mode can be easily observed visually and is recorded in the I-V characteristics of Figs. 3(b) and 4(b).

The cathode was operated at negative high voltage with the anode grounded. The precise position of the anode is not critical as long as the distance between electrodes is kept larger than the cathode dark space. If the anode is not in the direct beam path, a positive column develops to connect it to the electron beam created plasma. In the single gun experiments, the anode was a water-cooled copper tube $1.6-\mathrm{cm}$ in diameter placed $3 \mathrm{~cm}$ from the cathode and parallel to the cathode slot. The vacuum container was pumped to $10^{-3}$ Torr and commercial grade argon or helium flows into the system through a needle valve.
Figures 3 and 4 show the voltage-current characteristics of the electron beam produced in $\mathrm{He}$ and Ar discharges respectively. As shown, multi-kilowatt electron beams are created on a dc basis. Figures 3 (c) and 3(b) correspond to slot widths of $7 \mathrm{~mm}$ and $5 \mathrm{~mm}$ respectively, while Fig. 3(a) shows the characteristics of a $2.5-\mathrm{mm}$ slotted gun. Also shown in Fig. 3(a) are results obtained from a slotless gun; that is, a cathode of the same dimensions as the others but without a slot. In Fig. 3(a) notice that for an extended range of pressure, both the slotless and slotted guns present the same impedance. This can be expected since for these pressures in $\mathrm{He}$, the hollow cathode sheath is much larger than the 2.5$\mathrm{mm}$ slot width, and in the absence of a plasma in the slot the gun behaves as in the slotless case. Figures 3 and 4 also indicate that as the slot width is increased, the onset of the hollow cathode effect in the slot, which limits beam operation as described above, occurs under different discharge conditions.

The transition from the high impedance mode, in which the electron beam is produced, to a low impedance mode where the beam is extinguished, limits the maximum beam power generated. It is noteworthy this does not occur for the slotless guns of Fig. 3(a). Hence, the maximum beam power 


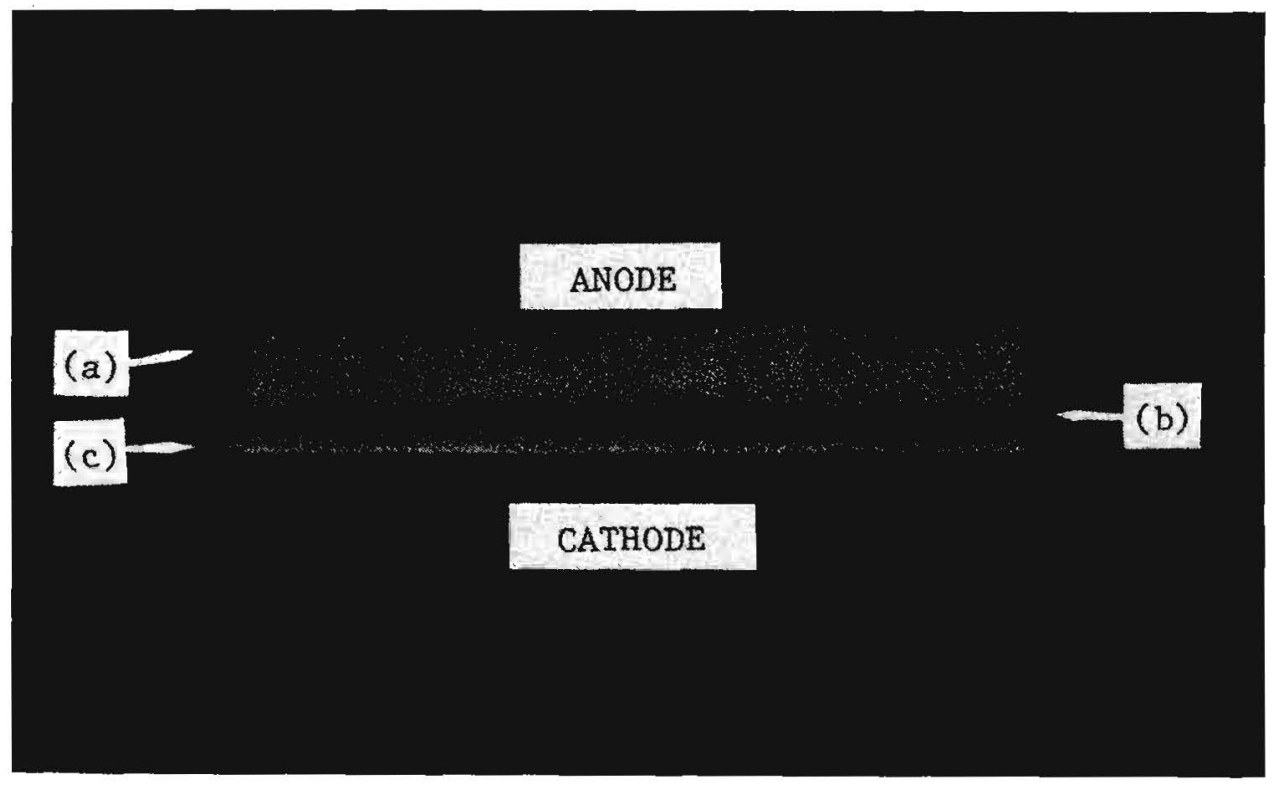

FIG. 2. Electron beam discharge in helium. The negative glow (a), the external dark space (b), and the cathode glow (c) are all shown.

obtained with the slotless gun is larger than that obtained from slotted guns. The transition region from the high-impedance beam mode to the low-impedance glow mode occurs at the maximum V-I point in all curves of Figs. 3 and 4; but this is illustrated only in Figs. 3(b) and 4(b) by the sharp movement of the $\mathrm{V}-\mathrm{I}$ characteristic.

The range of $\mathrm{Pd}$ in which maximum beam generation occurs is given in Fig. 5 for various slot widths. The data in Fig. 5 were taken from Figs. 3 and 4 with the $y$ axis being the maximum discharge power in the beam mode. That is the maximum power $\mathrm{V} \times \mathrm{I}$ that is achievable before the discharge mode transition occurs as shown in Figs. 3(b) and 4(b). Data for the $2.5-\mathrm{mm}$ gun in helium are not included in Fig. 5, because this gun behaves roughly as slotless [see Fig. 3(a)]. This is due to the fact that in helium, under the operating conditions of this gun, the dark space is thicker than the slot width, therefore the value $d=2.5 \mathrm{~mm}$ becomes meaningless, and cannot be used in the Pd plots of Fig. 5. Qualitatively, the maximum discharge power and the optimum $\mathrm{Pd}$ are larger for electron beams generated in helium as compared to those in argon. It is also evident that beam generation occurs over a larger $\mathrm{Pd}$ region for helium discharges as compared to argon.

\section{EFFICIENT ENERGY TRANSFER INTO PLASMA EXCITATION}

In applications in which the electron beam is to be used to create highly ionized plasmas, efficient deposition of the beam energy into the gas is requried. To efficiently create a plasma of smaller dimensions than the reaching distance $\ell$ of a kilovolt electron beam (e.g., $\ell=30 \mathrm{~cm}$ for helium at 1 Torr) the beam must be trapped or confined and not allowed to lose its energy in collisions with the walls. Trapping the electron beam would also considerably increase the effective current density in that region if the electron beam could be made to fold over on itself.

A configuration like the one shown in Fig. 1(b) has been used to partially confine the electron beam and limit the energy deposition to a fixed volume located between the guns. In this scheme, two transverse plasma guns are arranged facing each other. The distance between the front surfaces of the guns must be longer than $2 D$, where $D$ is the external dark space thickness, or the two guns will work as a single hollow cathode. The electron beam produced by one of the cathodes loses part of its energy in exciting and ionizing collisions as it travels towards the opposite gun and is then reflected by the electric fields in the opposite dark space. The process is similar to the one occurring in a slotted hollow cathode. ${ }^{7}$ In this configuration, we believe therefore that the electron beam would be electrostatically confined between the electron guns. The confinement is expected to be only partial, since a portion of the beam will be lost by scattering. The trapped beam creates a bright plasma with a sharp boundary that is clearly visible as shown in Fig. 1(c). This is qualitative evidence for a trapped beam. The electron beam current density and power density achieved with the trapping scheme is expected to be larger than the one obtained in the case of the untrapped beam. Further qualitative evidence for trapping is given below by comparing $\mathrm{I}-\mathrm{V}$ curves.

The voltage-current characteristics of our twin cathode design have been measured and the results are compared to single gun characteristics in Figs. 6(a) and 6(b) for Ar and $\mathrm{He}$ discharges respectively. The two cathodes employed were of identical structures each having a slot $200-\mathrm{mm}$ long and 5$\mathrm{mm}$ wide. The front walls had a radius of curvature of $15 \mathrm{~mm}$ and are separated from each other by $30 \mathrm{~mm}$. Figure $6(\mathrm{~b})$ displays the operating characteristics of one of these cathodes when the opposing electrode is either anode, cathode, or floated electrically. Notice the decrease in the discharge impedance, in Fig. 6(b) as the opposing electrode changes from anode, to a floating electrode, and finally to a cathode. The discharge impedance for the twin cathode is less than that of the single cathode by a factor of 3.5-5. We believe 


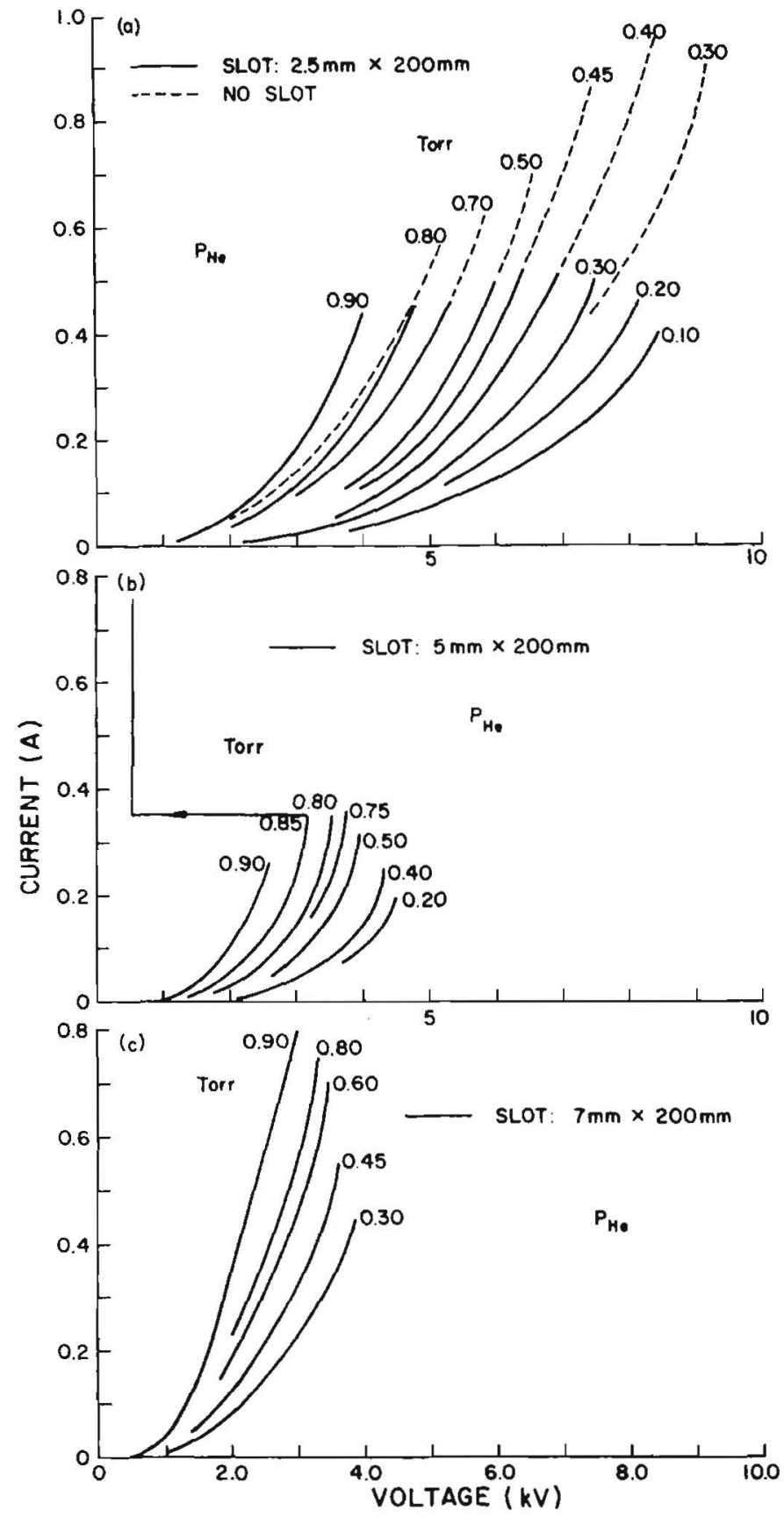

FIG. 3. I-V characteristics of the electron beam discharge in helium. The transition from the beam mode to the low impedance mode is only shown in 3 (b) for the 0.85 -Torr condition.

that an increase in the ionization rate, because of electron beam trapping, causes the decrease in discharge impedance as compared with the operation of a single cathode.

\section{ELECTRON BEAM PROFILES}

The electron beam profiles, in a single gun configuration, were measured using a movable thermocouple $1 \mathrm{~mm}$ in cross section to estimate the energy density profile of the electron beam. The thermocouple was placed at $2.0 \mathrm{~cm}$ from the cathode front wall and was moved perpendicular to the axis of the gun. The $2-\mathrm{cm}$ location is outside the external
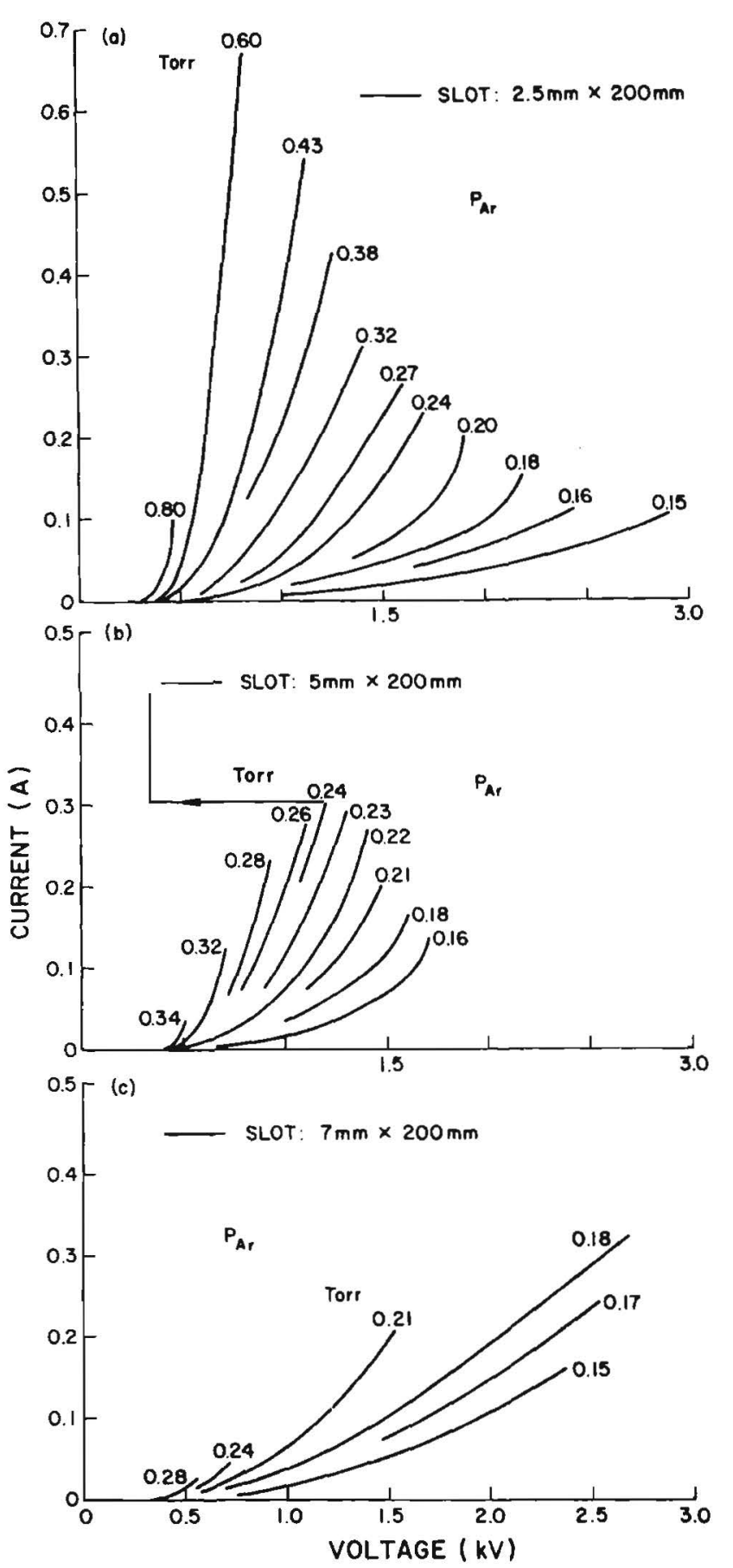

FIG. 4. I-V characteristics of the electron beam discharge in argon. The transition from the beam mode to the low impedance mode is only shown in 4 (b) for the 0.24 -Torr condition.

dark space and is within the range of interest for energy deposition discussed in Sec. III. Since the thermocouple temperature never exceeds $200^{\circ} \mathrm{C}$, conduction cooling dominates and we can, to a first approximation, relate the thermocouple temperature to the electron beam density. These measurements are approximate and give a qualitative idea of the beam profiles. The operating pressures under which the beam profile measurements were made varied from 0.5 to 1 Torr. The actual profiles are probably narrow- 


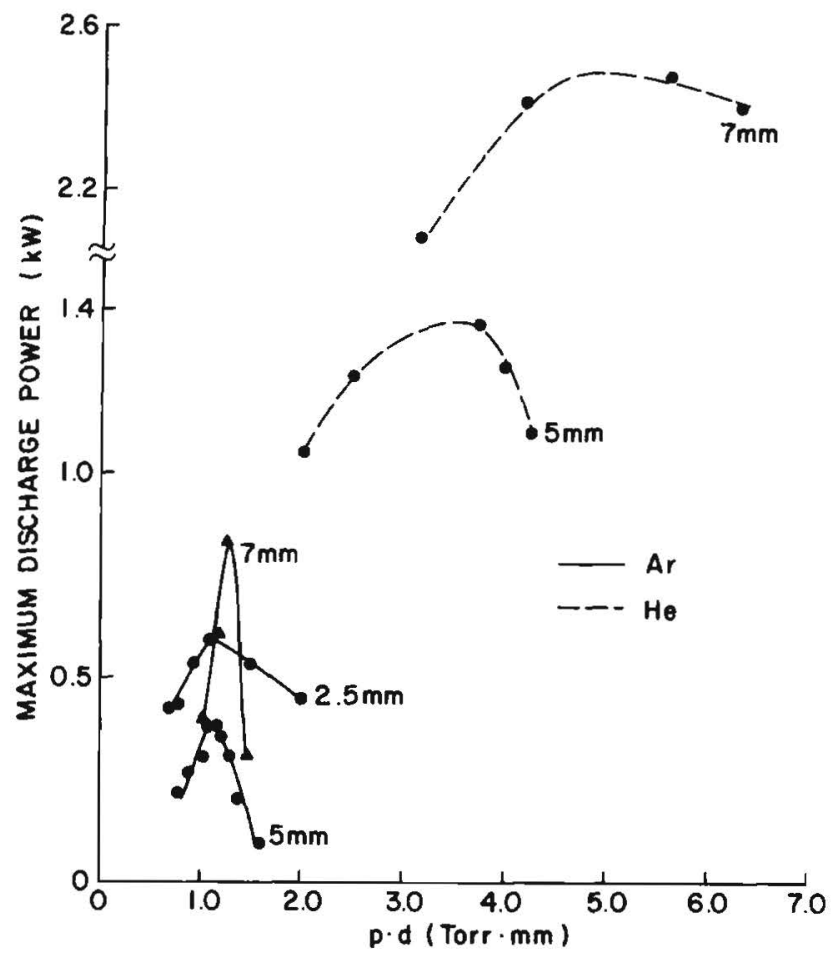

FIG. 5. Maximum discharge power, in the beam mode, plotted vs the operating parameter $P d$, where $P$ is the operating pressure and $d$ is the slot width $(2.5,5$, and $7 \mathrm{~mm})$.

er, since the thermocouple is not only heated by beam electrons, but also by collisions of other particles of the electron beam created plasma.

The beam profiles for argon and helium discharges corresponding to different cathode slots are shown in Fig. 7(a). We observe that for the 2.5 -mm-wide slot, the contribution of electrons from the front wall is larger and the beam profile is wider as compared to the better collimated beam that was obtained from the 5- and 7-mm slots in He. The beam profiles for a 5-mm slot were similar for both $\mathrm{He}$ and Ar discharges as shown in Fig. 7(a).

To enhance the electron beam current density in the region $2 \mathrm{~cm}$ from the cathode face we attempted to electrostatically focus the electron beam by changing the cathode front wall from flat to concave. This geometry is shown in Fig. $7(b)$ along with the measured beam profiles achieved with each cathode face. One can see that a better collimated beam is obtained with the concave nose, because of the focusing effect of the curved dark space. The thermocouple measurements indicate that we can create a $12-\mathrm{mm}$ wide beam at $2.0 \mathrm{~cm}$ from the cathode front wall.

\section{PULSED BEHAVIOR}

Electron guns with a 5-mm-wide slot were also operated in a pulsed electron beam mode to determine the peak beam current we could achieve. A $0.1-\mu \mathrm{F}$ capacitor was discharged across the electron beam glow discharge by switching to ground, a grounded grid thyratron at frequencies 1-20 Hz. The discharge voltage was controlled by changing the voltage of the dc power supply used to charge the capacitor.

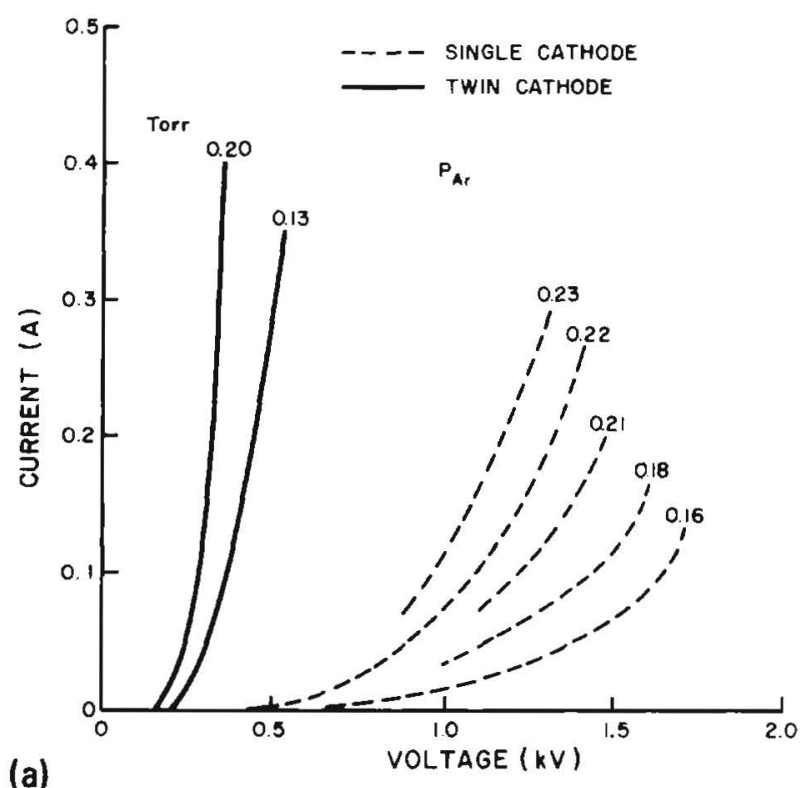

(a)

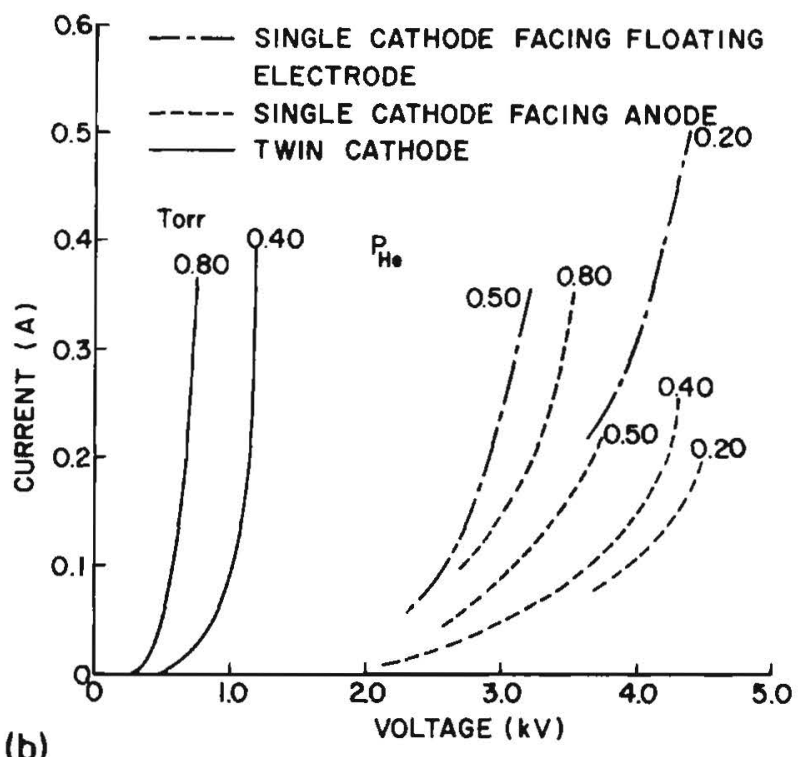

FIG. 6. (a) V-I characteristics in argon of the twin cathode configuration compared to the single cathode configuration. (b) V-I characteristics in helium are compared for three different electrode configurations.

The electron beam current pulses obtained with this configuration were $2-\mu$ s wide. The current and voltage were measured using high-frequency response probes connected to a Tektronix 7834 storage oscilloscope. A time lag 2-15 $\mu$ s can be observed between the moment at which the voltage pulse is applied across the electrodes and the buildup of the current pulse. The lag time is shorter in the twin cathode configuration than in the single cathode configuration for the same voltage and pressure. In both configurations the time lag decreases as voltage increases. As the pressure is increased, the time lag is also reduced. The peak cathode current as a function of the voltage measured between the cathode and anode is shown in Fig. 8(a). It is important to realize that the current and voltage of Fig. 8(a) correspond to the peak current and the corresponding voltage. That is the volt- 


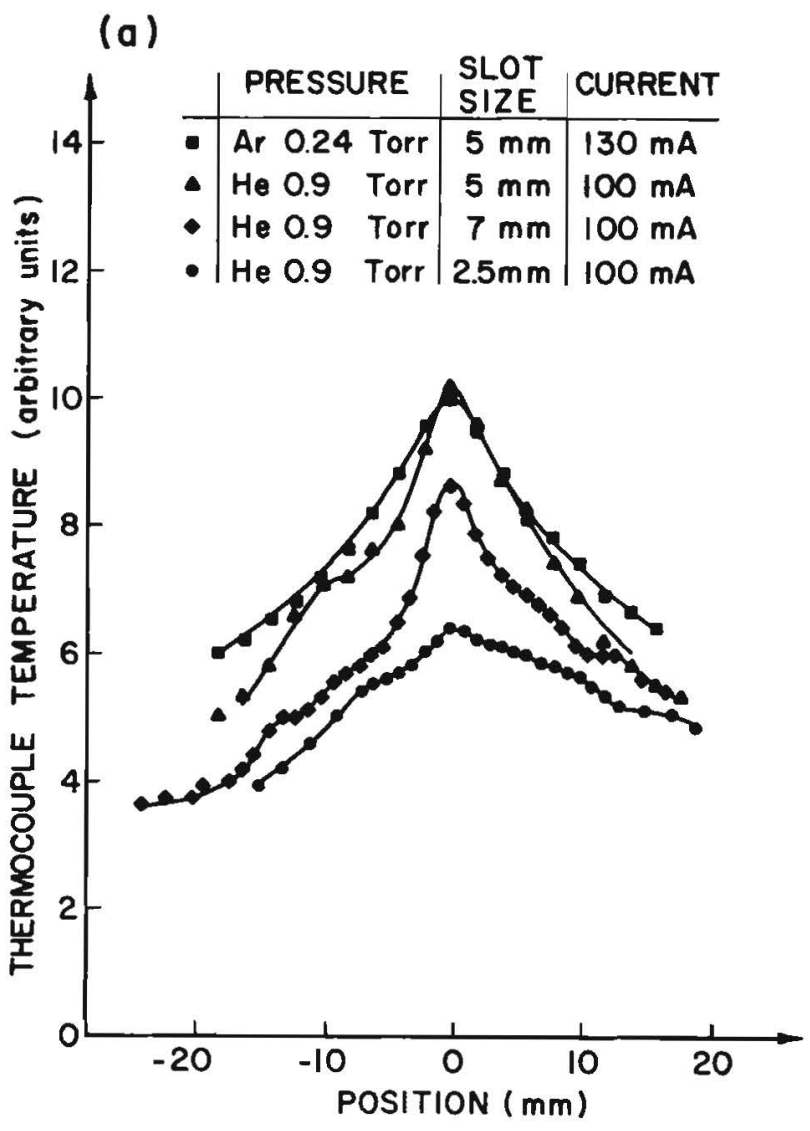

(b)

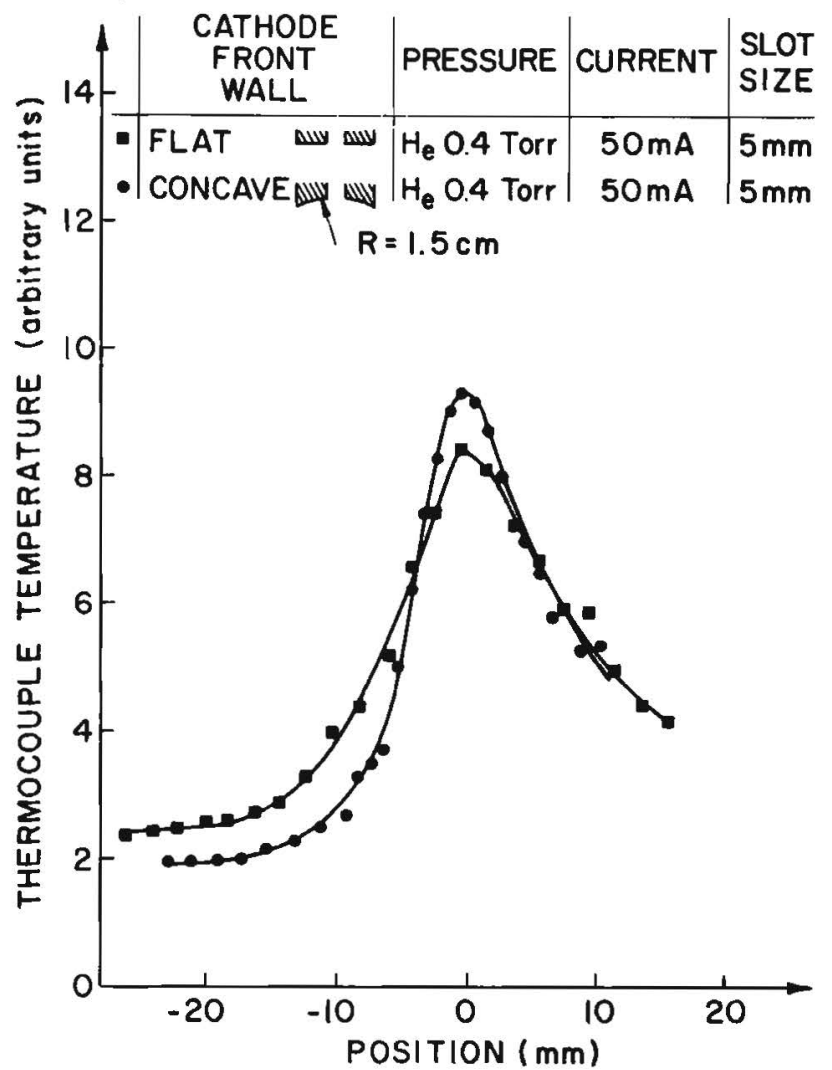

FIG. 7. Relative thermocouple temperature as a function of position within the electron beam discharge.
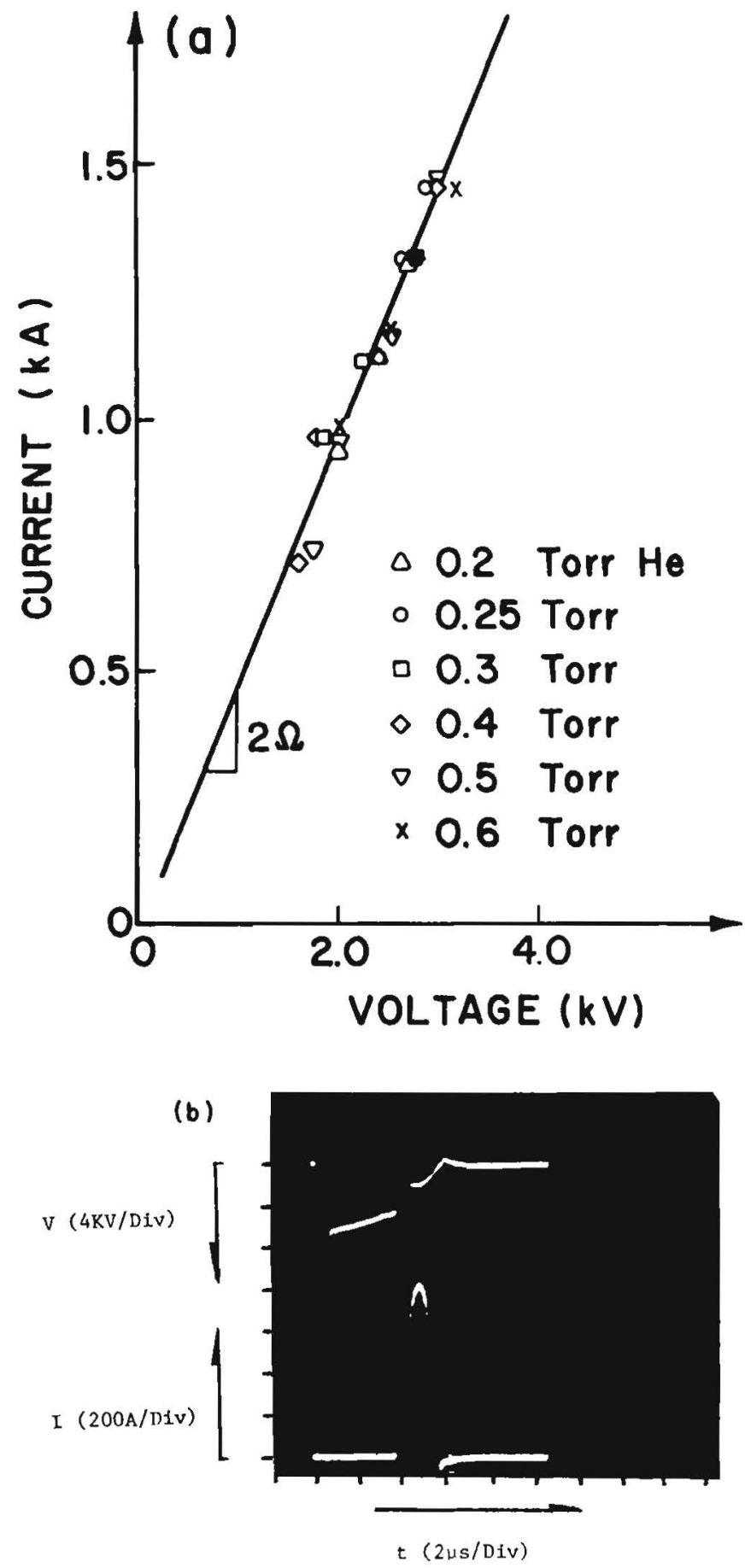

FIG. 8. (a) Peak cathode current versus anode-cathode voltage under pulsed $(2-\mu \mathrm{s}$-wide $)$ conditions. (b) Pulsed voltage and current as a function of time.

age measured at the time of peak current. See Fig. 8(b). The measured cathode current is the sum of the electron beam current emitted by the cathode and the ion current to the cathode.

To verify that a large electron beam current is produced in this pulsed glow discharge, Faraday probe measurements were taken using the twin electrode configuration. In this case, one of the cathodes was used as a Faraday probe in the 
(a)
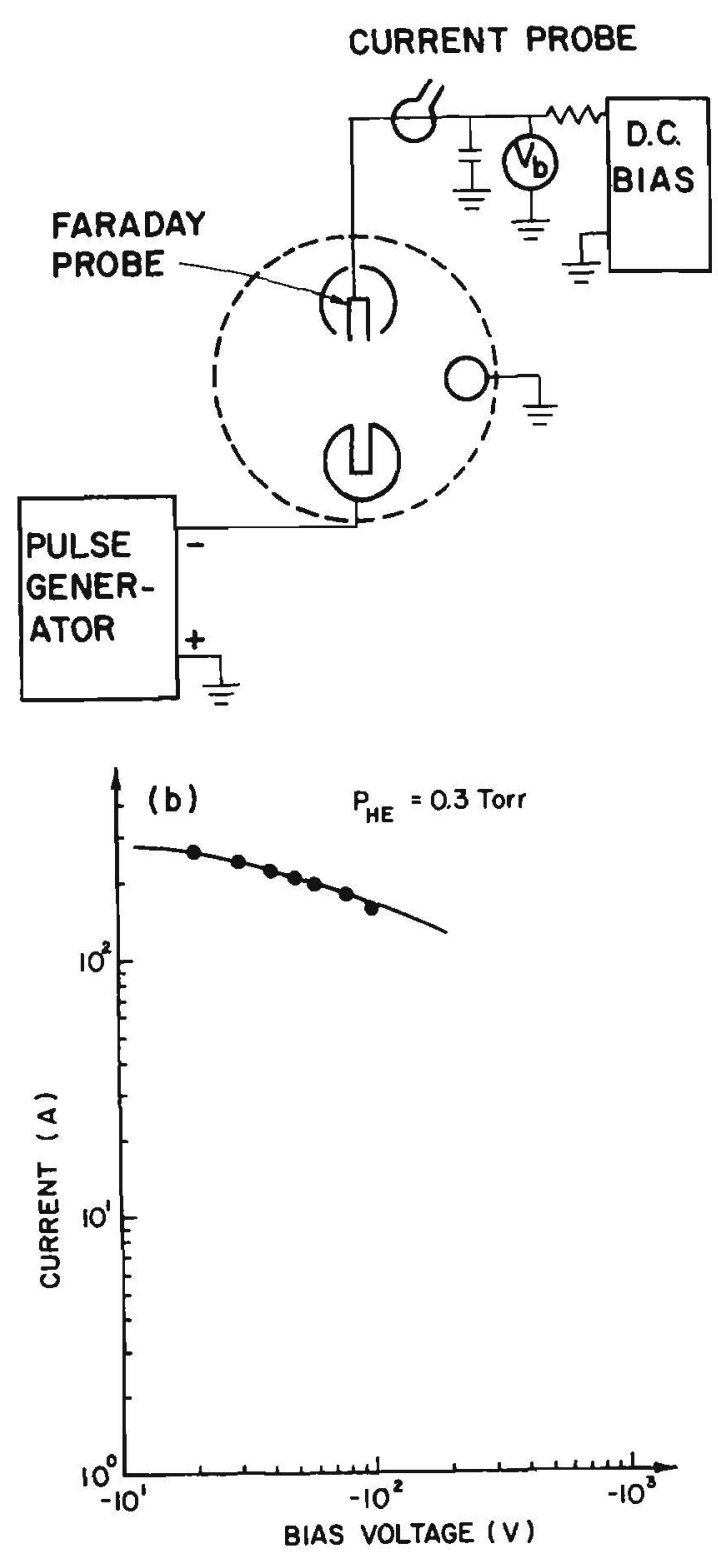

FIG. 9. (a) Faraday probe test apparatus for measuring the beam current under pulsed conditions, (b) Faraday probe current vs. repulsive bias voltage. setup shown in Fig. 9(a), where a negative bias voltage to the Faraday probe is supplied by a dc power supply.

The measured Faraday probe current is the sum of the beam electrons arriving at the probe and the ion current. Assuming that the ions arrive at the plasma sheath with the Bohm velocity, we have estimated that the ion current to the probe is small compared to the measured current. Also the Faraday probe will not collect all of the beam electrons for geometrical reasons, hence, the actual electron beam current produced by the electron gun may be larger than the one measured with the Faraday probe. Figure 9 (b) shows that more than $100 \mathrm{~A}$ of $100-\mathrm{eV}$ electrons have been experimentally verified to arrive at the probe under pulsed conditions.

\section{SUMMARY}

Transverse electron guns that operate at pressures up to 1 Torr have been developed without differential pumping. Kilowatt electron beams have been generated at currents up to $0.8 \mathrm{~A}$ (at $>2.5 \mathrm{kV}$ ) on a dc basis. Pulsed beam currents over $100 \mathrm{~A}$ of $>100 \mathrm{~V}$ have been measured, for pulses of $2-\mu \mathrm{s}$ duration, using a Faraday probe negatively biased at $100 \mathrm{~V}$. A trapped electron beam configuration is suggested for efficient deposition of the beam energy into a gaseous medium.

\section{ACKNOWLEDGMENTS}

This work was supported by the National Science Foundation. The early portion of these studies were supported by AFOSR. We wish to express our appreciation to W. K. Schuebel, C. P. Christensen, and Karl Persson for their encouragement and technical discussions.

'K. L. Boring and L. A. Stauffer, "A New Non-Thermoionic Electron Gun," National Electronic Conference Proceedings (1963) Vol. 19, 535.

${ }^{2}$ J. W. Davis, E. A. Pinsley, and A. P. Walch, United Aircraft Research Laboratories, Report UAR-E66, (1966).

${ }^{3}$ J. J. Rocca, J. Meyer, Z. Yu, and G. J. Collins, in Thirty-fourth Gaseous Electronic Conference, Boston, October 1981.

${ }^{4}$ N. J. Lanno, J. T. Verdeyen, S. S. Chan, and B. G. Streetman, Appl. Phys. Lett. 39, 622 (1981).

${ }^{5}$ H. S. W. Massey, Electronic and Ionic Impact Phenomena, (Oxford University, London, 1971] Vol. I.

${ }^{6} \mathrm{G}$. Carter and J. S. Collison, Ion Bombardment of Solids, (American Elsevier, New York, 1968).

${ }^{7} \mathrm{C}$. W. Willet, Introduction to Gas Lasers: Population Inversion Mechanisms, (Pergamon, New York, 1974). 